\section{Clinical Excellence in Graduate Nursing Education: A Case Study of Information Technology Solutions}

\section{Dr. Lauren St. John* DNP, RN, FNP-BC, MCHC}

Associate Chair for Clinical Education \& Clinical Assistant Professor The University of Texas at Arlington, Texas, USA fully met within the first year of implementation. Partially-met deliverables require additional time to be fully realized and evaluated. Data collection is planned to continue for observing results of the software implementation over time, particularly as refinements progress and the software is fully adopted by all intended users.

Keywords: Clinical practicum; Graduate nursing education; Information technology; Preceptorship

\section{Introduction}

The role of information technology in managing clinical education is not well-studied in graduate nursing programs, yet organized documentation for clinical activities is necessary for programs to efficiently demonstrate alignment with professional nursing standards and guidelines [1]. Nursing and allied health programs internationally are looking for innovative methods to solve prevalent clinical education and placement issues, challenging the one-on-one model for student-preceptor arrangements and working to contribute evidence to support effective methods for graduate clinical education [2-5].

This case study describes the context leading one graduate nursing department to select and implement a web-based clinical management system ("the software"). Transition details and project deliverables are presented. Results support the conclusion that innovative software may allow programs to expedite clinical placement while promoting student success, eliminating cost and generating objective evidence to enrich clinical education.

\section{Background}

The graduate programs of the university discussed include online and campus versions of seven Nurse Practitioner specialties, an online Nursing Education program, and online/campus versions of a Doctor of Nursing Practice program. For academic year 2017-2018, approximately 2,500 clinical placements within the graduate programs were confirmed. One clinical placement is defined as one unique student in one unique clinical/practicum course requiring preceptorship. Many placements included more than one preceptor and/or facility arrangement.

Prior to implementing the software, the graduate programs had siloed clinical documentation stored among five electronic systems. Clinical coordination workflow included excess manual data entry and student communication regarding practicum requirements and placement concerns. Storage space and structure of the existing systems proved to be insufficient for the growing volume of students and placements. Consequently, potential for error existed with implications impacting student progression and financial aid. Situations like these can lead to student distress and loss of focus from education.

It was the universal consensus of leadership and personnel involved in clinical management that the graduate department had optimized clinical education processes and documentation potential with the resources in use and available within the university. A vision statement to focus the search for a software solution was drafted, to have a single management system for documenting clinical education 
that would seamlessly guide students from admission to graduation. This system was to be a long-term solution promoting success of the graduate programs and enabling the scalability requisite for expansion into other states.

\section{Selecting a Software System}

Thorough market research of software designed to manage clinical education was conducted over approximately 2 years. Demonstrations were presented to faculty and staff by sales representatives of several well-established clinical management software companies. Additional sessions were organized with consultants and development specialists of the systems to discuss, with meticulous detail, the critical features and software potential relative to clinical education and student placement coordination.

Leadership involved in software selection prepared an executive summary of the research, comparing available software options in key deliverable areas of usability, flexibility, cost elimination, manual labor reduction, efficiency and other software uses apart from clinical data management. Despite the fact that many products advertised ability to perform similar functions and would replace the same systems currently in use by the graduate department, several objective differences between the potential systems were noted:

- Single step vs. multiple steps to accomplish equivalent functions;

- User-friendly, clear navigation vs. clarity issues in navigating single processes;

- Extent of configuration potential and software flexibility;

- Effect of configurations on ability to maintain functionality of other software features;

- Potential increase in manual labor resulting from above-mentioned limitations.

\section{Anticipated Project Deliverables}

A contractual agreement was finalized with a web-based clinical management software company in accordance with the executive summary recommendation. Anticipated deliverables of full implementation included evidence of efficient clinical coordination; increased student volume managed successfully by clinical placement staff; a clear student placement process; ability to manage individual site/state requirements and affiliation agreement terms; centralized location for clinical documentation supporting program accreditation; streamlined methods for evaluating student clinical performance; ample reporting capabilities; and opportunities for improved communication among the entire educational team.

\section{Implementation Process}

Software implementation began with scoping sessions designed to aid the software consultants in understanding the present data management methods for graduate clinical education. A project lead from the graduate nursing department was selected to oversee the software configuration and be the main point of contact for the software consultant team. Organized planning resulted in a timeline for project implementation prioritized according to urgency, complexity, and windows of opportunity for transitioning away from legacy systems with minimal disruption to current operations.

\section{System Configuration and Deployment}

In the eight months following project scoping, sections of the software were systematically configured and deployed for major uses in this order: (1) program-specific logbooks for student-reported practicum data; (2) student records system integration for degree program and course enrollment; (3) communication templates and associated student clinical performance evaluations; (4) agency affiliation agreement database with parent-child hierarchy; (5) program-specific student self-placement submissions and preceptor/clinical site vetting process; (6) custom fields and tracking within the self-placement wizard of the software for streamlined placement coordination; and (7) custom alerts and reports for validating state authorization, accreditation, and other compliance markers.

Refinement of the software to optimize features for the graduate programs remains an ongoing commitment and contractual agreement of the software consultants. Undergraduate nursing and public health programs in the university have also adopted the software, creating college-wide alignment potential within clinical programs.

\section{Theoretical Approach to Change}

Leadership skills to guide this change were critical for successful implementation of the software. Concepts and theoretical constructs on the diffusion of innovations prepared the project lead with tools for facilitating adoption of new technology among students, faculty, and staff. Familiarity with stages of the innovation-decision process and attributes of innovations such as relative advantage, compatibility, complexity, trialability, and observability played an important role in executing this transition [6].

\section{Observable Results}

Throughout his project, leadership returned to anticipated deliverables for a structured approach to objectively evaluate results of the software implementation. Due to distinct differences in features and workflow of legacy systems and the software, many deliverables do not have a quantitative pre-implementation and post-implementation relationship suitable for analysis. Rather, observable results of this case study are reflected in the presence or absence of challenges related to clinical data management observed with legacy systems and again observed with the software. Fully-functioning, practical features of the software not possible with legacy systems are also observable.

The results of this case study reflect implementation of the software to a limited degree as completed during the first eight months of configuration and deployment. Cumulative developments are anticipated over time, warranting future data collection to evaluate longer-term outcomes of the software on clinical placement management within graduate nursing programs.

\section{Usability/Flexibility}

Usability of the software was evaluated as a quality metric measured by success rate of student self-placement submissions, clinical clearance, and execution of intended software functions by role-specific users. Within hours of deploying the self-placement submission process to students, more than twenty had successfully logged in and completed self-placement submissions for an upcoming clinical or 
practicum course, exceeding average numbers of submissions completed in a twenty-four hour period using established legacy systems.

Within three months following deployment of the new self-placement process, more than eight hundred student placements were successfully vetted according to programmatic preceptor and site requirements and processed to full clinical clearance, including timely execution of affiliation agreements. Unsolicited student reports of subjective satisfaction with the software and redesigned clinical coordination process were also received. Comprehensive data collection to evaluate usability and other outcome areas is planned as a longer-term longitudinal study of the software implementation.

In the eight months following deployment of student logbooks and evaluation features, all users successfully carried out functions specific to their role using the software. Evaluations were sent through the software to preceptors, students, and placement supervisors for evaluating clinical performance, site appropriateness, and quality of the educational experience. Students successfully logged timesheets, patient encounters, and other clinical/practicum activities as anticipated, and faculty reviewed and provided feedback on student logbook records.

\section{Cost Elimination}

The financial investment per student license for the software is approximately $80 \%$ of the per student cost of one legacy system. Additionally, four other systems were eliminated from clinical management operations and corresponding license fees were also eliminated As time continues and the anticipated trajectory of the software outcomes is confirmed, it will become possible to quantify the number of students that each clinical coordination staff member manages to successful clinical/practicum clearance.

An observation at two months following deployment of the self-placement arm of the software demonstrates improvements in clinical coordination efficiency. The software allowed clinical placement staff to proactively clear students for clinical with an additiona 3 months between the usual clearance date and the clinical start date. This time is anticipated to continue increasing, resulting in complete clinical clearances for a majority of students many months in advance of each clinical start date. This increase is directly attributable to streamlined documentation requirements and flow of clinical clearance processes for students and staff alike, generating time resources for clinical coordination staff to support students in identifying and securing placements appropriate for meeting course and program objectives.

\section{Manual Labor Reduction/Efficiency}

Several features of the software that were not possible with legacy systems concretely reduce manual administrative work with streamlined processes resulting in greater efficiency. One example is the observable integration of the software with the university student records system. This integration has successfully allowed enrollment data, student profile information, degree plan projections, and course outcomes to feed directly into the software on a nightly basis. Manual lookup of degree plan data and maintenance of accurate projected enrollment lists for clinical clearance is no longer necessary.

Students submit self-placement requests through the software via automatically generated links that align and update with course numbers and start dates for projected and actual enrollment. Custom self-placement forms and a wizard tool allow the clinical coordination staff to accurately review student placement submissions on any number of predefined qualifications, comparing the details of the submission to preceptor/course requirements established by graduate nursing program directors.

Agency affiliation agreements are stored in the software and student placement submissions are filed within the existing database of affiliated clinical facilities. Site-specific requirements and unique affiliation agreement terms noted by the contracts or coordination teams are viewable at the time of self-placement submission, promoting completion and compliance for every placement. The parent-child hierarchy of affiliations allows immediate allocation of a placement to the correct legal agreement and site.

Students are kept informed of their clinical clearance status in real-time via communication that occurs within the clinical placement record in the software. The correspondence related to each placement is thereby contained within a single source and has reduced the volume of email communication between students and coordinators regarding placement status updates. Customized reports and alerts prompt validation of compliance requirements and purification of the database from information duplicates, errors and omissions.

\section{Additional System Uses}

Access to the software is possible through external accounts or internal users via the university single sign on. Combined with the ability to set custom permissions and custom user roles, it is now possible for the entire educational team to collaborate and communicate within the software that connects student profiles and comprehensive placement data. The university is exploring opportunities for human resources, admissions/advising, preceptors, faculty, administrators, and additional university support personnel to connect in innovative ways to enrich the student educational experience through the software.

Custom reporting capabilities of the software enable any clinical placement data to be compared with any data from the student records system. This fact indicates potential for cutting-edge clinical education research and outcomes analysis that is made possible by the flexible capabilities to view actual clinical education data in nearly any arrangement or combination of variables. Whether the desire is to compare student evaluation results with preceptor credentials, clinical site and preceptor attributes with student retention rates, or any other construction of analysis relative to clinical preceptorship and the student educational record, it is all possible.

\section{Overall Effectiveness of the Software System}

This information technology solution for clinical placement management in graduate nursing education has demonstrated real-world effectiveness in all anticipated deliverables. Clinical placement records are organized within a single, user-friendly and flexible system that has eliminated cost, reduced manual labor, expedited processes and accommodated additional software uses apart from clinical placement management.

\section{Conclusion and Future Considerations}

Information technology designed for clinical management may bring timely solutions for graduate nursing programs experiencing growth as well as programs of any size desiring quality improvement through robust clinical process-outcome evaluations into the future. 
Just as the Centers for Medicare and Medicaid Services are dedicated to improving interoperability and encouraging the demonstration of meaningful use of electronic health records in health care delivery, nursing education leadership may begin to consider what constitutes meaningful use of information technology in student placement processes and delivery of quality clinical education [7]. The results of this case study show that it is possible with innovative software to examine detailed correlations between clinical placement characteristics and student success metrics. Real student experiences, real education methods, and real clinical education outcomes may all be enriched to impact real patients, real healthcare problems, and real lives on the basis of evidence that is able to be generated through the software capabilities.

\section{Acknowledgment}

The author would like to formally thank Dr. Kathryn Daniel, Associate Professor at the University of Texas at Arlington, for initiating the market research on clinical management software systems and providing significant contributions during the evaluation and selection of the information technology discussed in this case study.

\section{Financial Support}

This research did not receive any specific grant from funding agencies in the public, commercial, or not-for-profit sectors.

\section{References}

1. Commission on Collegiate Nursing Education (2018) Standards for Accreditation of Baccalaureate and Graduate Nursing Programs. Commission on Collegiate Nursing Education, USA.

2. Clark CA, Kent KA, Riesner SA (2018) A new approach for solving an old problem in Nurse Practitioner Clinical Education. The Journal for Nurse Practitioners 14: 69-75.

3. Drayton-Brooks SM, Gray PA, Turner NP, Newland JA (2017) Building clinical education training capacity in nurse practitioner programs. J Prof Nurs 33: 422-428.

4. Fairbrother M, Nicole M, Blackford J, Nagarajan SV, McAllister L (2016) A New Model of Clinical Education to Increase Student Placement Availability: The Capacity Development Facilitator Model. Asia-Pacific Journal of Cooperative Education 17: 45-59.

5. Romig BD, Tucker AW, Hewitt AM, O’Sullivan Maillet J (2017) The future of clinical education: Opportunities and challenges from allied health Deans' perspective. J Allied Health 46: 43-55.

6. Rogers E (2003) Diffusion of Innovations (5thedn). Simon and Schuster, New York, USA. Pg no: 576.

7. Centers for Medicare and Medicaid Services (2018) Promoting Interoperability. Centers for Medicare and Medicaid Services, Maryland, USA. 


\section{dit \\ HERALD}

Journal of Anesthesia \& Clinical Care

Journal of Addiction \& Addictive Disorders

Advances in Microbiology Research

Advances in Industrial Biotechnology

Journal of Agronomy \& Agricultural Science

Journal of AIDS Clinical Research \& STDs

Journal of Alcoholism, Drug Abuse \& Substance Dependence

Journal of Allergy Disorders \& Therapy

Journal of Alternative, Complementary \& Integrative Medicine

Journal of Alzheimer's \& Neurodegenerative Diseases

Journal of Angiology \& Vascular Surgery

Journal of Animal Research \& Veterinary Science

Archives of Zoological Studies

Archives of Urology

Journal of Atmospheric \& Earth-Sciences

Journal of Aquaculture \& Fisheries

Journal of Biotech Research \& Biochemistry

Journal of Brain \& Neuroscience Research

Journal of Cancer Biology \& Treatment

Journal of Cardiology: Study \& Research

Journal of Cell Biology \& Cell Metabolism

Journal of Clinical Dermatology \& Therapy

Journal of Clinical Immunology \& Immunotherapy

Journal of Clinical Studies \& Medical Case Reports

Journal of Community Medicine \& Public Health Care

Current Trends: Medical \& Biological Engineering

Journal of Cytology \& Tissue Biology

Journal of Dentistry: Oral Health \& Cosmesis

Journal of Diabetes \& Metabolic Disorders

Journal of Dairy Research \& Technology

Journal of Emergency Medicine Trauma \& Surgical Care

Journal of Environmental Science: Current Research

Journal of Food Science \& Nutrition

Journal of Forensic, Legal \& Investigative Sciences

Journal of Gastroenterology \& Hepatology Research

Journal of Gerontology \& Geriatric Medicine

Journal of Genetics \& Genomic Sciences

Journal of Hematology, Blood Transfusion \& Disorders

Journal of Human Endocrinology

Journal of Hospice \& Palliative Medical Care

Journal of Internal Medicine \& Primary Healthcare

Journal of Infectious \& Non Infectious Diseases

Journal of Light \& Laser: Current Trends

Journal of Modern Chemical Sciences

Journal of Medicine: Study \& Research

Journal of Nanotechnology: Nanomedicine \& Nanobiotechnology

Journal of Neonatology \& Clinical Pediatrics

Journal of Nephrology \& Renal Therapy

Journal of Non Invasive Vascular Investigation

Journal of Nuclear Medicine, Radiology \& Radiation Therapy

Journal of Obesity \& Weight Loss

Journal of Orthopedic Research \& Physiotherapy

Journal of Otolaryngology, Head \& Neck Surgery

Journal of Protein Research \& Bioinformatics

Journal of Pathology Clinical \& Medical Research

Journal of Pharmacology, Pharmaceutics \& Pharmacovigilance

Journal of Physical Medicine, Rehabilitation \& Disabilities

Journal of Plant Science: Current Research

Journal of Psychiatry, Depression \& Anxiety

Journal of Pulmonary Medicine \& Respiratory Research

Journal of Practical \& Professional Nursing

Journal of Reproductive Medicine, Gynaecology \& Obstetrics

Journal of Stem Cells Research, Development \& Therapy

Journal of Surgery: Current Trends \& Innovations

Journal of Toxicology: Current Research

Journal of Translational Science and Research

Trends in Anatomy \& Physiology

Journal of Vaccines Research \& Vaccination

Journal of Virology \& Antivirals

Archives of Surgery and Surgical Education

Sports Medicine and Injury Care Journal

International Journal of Case Reports and Therapeutic Studies

Submit Your Manuscript: http://www.heraldopenaccess.us/Online-Submission.php 